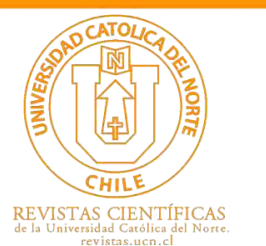

\title{
Edge irregularity strength of certain families of comb graph
}

\author{
Xiujun Zhang ${ }^{1}$ (D) orcid.org/0000-0002-6704-031X \\ Murat Cancan $^{2}$ (1) orcid.org/0000-0002-8606-2274 \\ Muhammad Faisal Nadeem ${ }^{3}$ (D) orcid.org/0000-0002-3175-7191 \\ Muhammad Imran ${ }^{4}$ (1) orcid.org/0000-0002-2272-6342 \\ ${ }^{1}$ Chengdu University, School of Information Science and Engineering, Chengdu, China. \\ woodszhang@cdu.edu.cn \\ ${ }^{2}$ Van Yznc Yil University, Faculty of Education, Van, Turkey. \\ m_cencen@yahoo.com \\ ${ }^{3}$ COMSATS University Islamabad, Dept. of Mathematics, Lahore, Pakistan. \\ mfaisalnadeem@ymail.com \\ ${ }^{4}$ COMSATS University Islamabad, Dept. of Mathematics, Lahore, Pakistan. \\ imranbepakistani@gmail.com
}

Received: February 2020 | Accepted: May 2020

\section{Abstract:}

Edge irregular mapping or vertex mapping $h: V(U) \rightarrow\{1,2,3,4, \ldots, s\}$ is a mapping of vertices in such a way that all edges have distinct weights. We evaluate weight of any edge by using equation $w t_{h}(c d)=h(c)+h(d), \forall c, d \in$ $V(U)$ and $\forall c d \in E(U)$. Edge irregularity strength denoted by es $(U)$ is a minimum positive integer use to label vertices to form edge irregular labeling. In this paper, we find exact value of edge irregularity strength of different families of comb graph.

Keywords:Irregular assignment; Irregularity strength; Edge irregularity strength; Comb graphs.

$\operatorname{MSC}(2020): 05 C 78$.

\section{Cite this article as (IEEE citation style):}

X. Zhang, M. Cancan, M. F. Nadeem and M. Imran, "Edge irregularity strength of certain families of comb graph", Proyecciones (Antofagasta, On line), vol. 39, no. 4, pp. 787-797, Aug. 2020, doi 10.22199/issn.0717-6279-2020-04-0049.

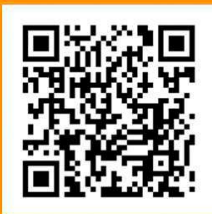

Article copyright: (c) 2020 Xiujun Zhang, Murat Cancan, Muhammad Faisal Nadeem, and Muhammad Imran. This is an open access article distributed under the terms of the Creative Commons License, which permits unrestricted use and distribution provided the original author and source are credited. 


\section{Introduction:}

In this paper, we consider finite, simple and undirected graphs. The procedure of assignment of numbers (either positive or negative) to the elements of a graph $G$ is termed as labeling. Vertex set $V(G)$ and edge set $E(G)$ are the elements of a graph $G$. If we label vertices or edges then this labeling is categorized as vertex labeling or edge labeling respectively. If we label both vertices and edges, then this labeling is termed as total labeling.

Chartrand et al.[13] had introduced edge labeling for a graph $G$. We call this labeling as irregular assignments because all vertices have distinct weights. Irregularity strength $s(G)$ is a minimum positive integer which is used to form irregular labeling. Results regarding irregularity strength can be seen in $[8,12,14,18]$

Vertex irregular mapping or edge mapping $h: E(G) \longrightarrow\{1,2,3,4, \ldots, s\}$ is a mapping of edges in such a way that all vertices have distinct weights. We evaluate weight of any vertex by using equation $w t_{h}(c)=\Sigma h(c d)$, $\forall c, d \in V(G)$ and $c d \in E(G)$. Motivated by Chartrand's work, Bača at $[10]$ introduced new labeling named as vertex irregular total labeling. Vertex irregular total labeling for a graph $G$ is a mapping $h: E(G) \cup V(G) \longrightarrow$ $\{1,2,3,4, \ldots, s\}$ in such a way that the total vertex weight is different for all vertices. We can evaluate total vertex weight by using the relation $w t_{h}(c)=h(c)+\Sigma h(c d), \forall c, d \in V(G)$ and $\forall c d \in E(G)$. Total vertex irregularity strength denoted by $\operatorname{tvs}(G)$ is a minimum positive integer use to label vertices to form vertex irregular total labeling. For more results on labeling and trees we refer to see $[1,2,4,5,9,11,15,16,17,19,20,21,22,24]$.

Both edge irregular and vertex irregular was a new labeling categorized as totally irregular total labeling developed by Marzuki in [10] by the motivation of previous improvements. Total irregularity strength for a graph $U$ is denoted as $t s(U)$. Results related to irregular total labeling were developed in $[10]$.

Because of provocation of preceding results Ahmed et. al in [2] developed a new concept of edge irregularity strength denoted by es $(U)$ which is a minimum positive integer use to label vertices to form edge irregular labeling. Inspired by this, more results were developed in $[5,6,23]$.

Theorem 1.1. [2] Let $G$ be a simple graph with maximum degree $\Delta=$ $\Delta(G)$. Then es $(G) \geq \max \{\lceil(|E(G)|+1) / 2\rceil, \Delta(G)\}$. 


\section{Comb Graph:}

Let us consider a path graph $P_{n}$, having $n \geq 1$ vertices and $(n-1)$ edges. The comb graph $C b_{n}$ is defined by $P_{n} \odot K_{1}$. It has $2 n$ vertices and $(2 n-1)$ edges.

\section{Comb Graph $C a_{n}$ :}

Comb graph $C a_{n}$ is formed by vertex set $V\left(C a_{n}\right)=\left\{a_{i}^{j} ; 1 \leq i \leq j+1,1 \leq\right.$ $j \leq n\}$ and edge set $E\left(C a_{n}\right)=\left\{a_{1}^{j} a_{1}^{j+1} ; 1 \leq j \leq n-1\right\} \bigcup\left\{a_{i}^{j} a_{i+1}^{j} ; 1 \leq j \leq n\right.$, $1 \leq i \leq j\}$. It has $\left(\frac{n^{2}+3 n}{2}\right)$ vertices and $\left(\frac{n^{2}+3 n-2}{2}\right)$ edges.

Theorem 3.1. Let $C a_{n}$ be a comb graph. Then es $\left(C a_{n}\right)=\left\lceil\frac{n(n+3)}{4}\right\rceil$

Proof. Let $C a_{n}$ be a comb graph. We have to show that es $\left(C a_{n}\right)$ $=\left\lceil\frac{n(n+3)}{4}\right\rceil$. From Theorem 1.1 we get lower bound $e s\left(C a_{n}\right) \geq\left\lceil\frac{n(n+3)}{4}\right\rceil$. For converse, we have to prove that $e s\left(C a_{n}\right) \leq\left\lceil\frac{n(n+3)}{4}\right\rceil$. For this define a labeling on vertex set such that

If $j$ is odd,

$$
f\left(a_{i}^{j}\right)= \begin{cases}\left\lceil\frac{i}{2}\right\rceil, & \text { if } j=1,1 \leq i \leq j+1 \\ \left\lceil\frac{j^{2}+3 j-2 i+2}{4}\right\rceil, & \text { if } j \equiv 3(\bmod 4), 1 \leq i \leq j \\ \left\lceil\frac{j^{2}+3 j-2 i+4}{4}\right\rceil-2, & \text { if } j \equiv 3(\bmod 4), i=j+1 \\ \left\lceil\frac{j^{2}+3 j-2 i+2}{4}\right\rceil, & \text { if } j \equiv 1(\bmod 4), 1 \leq i \leq j+1\end{cases}
$$

If $j$ is even,

$$
f\left(a_{i}^{j}\right)= \begin{cases}\left\lceil\frac{i+2}{2}\right\rceil, & \text { if } j=2,1 \leq i \leq j+1 \\ \left\lceil\frac{j^{2}+j+2 i-2}{4}\right\rceil, & \text { if } j>2,1 \leq i \leq j+1\end{cases}
$$

Now we evaluate weights for all edges as

$$
w_{t}\left(a_{1}^{j} a_{1}^{j+1}\right)= \begin{cases}\frac{j^{2}+3 j+2}{2}, & \text { if } \mathrm{j} \text { is odd } \\ \frac{j^{2}+3 j+4}{2}, & \text { if } j \equiv 2(\bmod 4) \\ \frac{j^{2}+3 j+2}{2}, & \text { if } j \equiv 4(\bmod 4)\end{cases}
$$


If $j$ is odd,

$$
w_{t}\left(a_{i}^{j} a_{i+1}^{j}\right)= \begin{cases}2, & \text { if } i=1, j=1 \\ \frac{j^{2}+3 j-2 i+2}{2}, & \text { if } j \equiv 3(\bmod 4), 1 \leq i \leq j-1 \\ \frac{j^{2}+3 j-2 i}{2}, & \text { if } j \equiv 3(\bmod 4), i=j \\ \frac{j^{2}+3 j-2 i+2}{2}, & \text { if } j \equiv 1(\bmod 4), 1 \leq i \leq j\end{cases}
$$

If $j$ is even,

$$
w_{t}\left(a_{i}^{j} a_{i+1}^{j}\right)=\frac{j^{2}+j+2 i}{2}, \text { if } 1 \leq i \leq j
$$

On the basis of above calculations we see that all edges have distinct weights.

Hence, $e s\left(C a_{n}\right)=\left\lceil\frac{n(n+3)}{4}\right\rceil$

\section{Comb Graph $C d_{n}$ :}

Comb graph $C d_{n}$, is formed by vertex set $V\left(C d_{n}\right)=\left\{a_{i}^{j} ; 1 \leq j \leq n, 1 \leq i \leq\right.$ $\left.\left\lfloor\frac{j+3}{2}\right\rfloor\right\}$ and edge set $E\left(C d_{n}\right)=\left\{a_{i}^{j} a_{i+1}^{j} ; 1 \leq j \leq n, 1 \leq i \leq\left\lfloor\frac{j+1}{2}\right\rfloor\right\} \cup\left\{a_{1}^{j} a_{1}^{j+1}\right.$; $1 \leq j \leq n-1\}$. It has $\left(\frac{n^{2}+6 n}{4}\right)$ vertices and $\left(\frac{n^{2}+6 n-4}{4}\right)$ edges.

Theorem 4.1. Let $C d_{n}$ be a comb graph. Then $e s\left(C d_{n}\right)=\frac{n^{2}+6 n}{8}$.

Proof. Let $C d_{n}$ be a comb graph. We have to show that $e s\left(C d_{n}\right)=$ $\frac{n^{2}+6 n}{8}$. From Theorem 1.1 we get lower bound $e s\left(C d_{n}\right) \geq \frac{n^{2}+6 n}{8}$. For converse, we have to prove that $e s\left(C a_{n}\right) \leq \frac{n^{2}+6 n}{8}$.. For this define a labeling on vertex set such that

$$
f\left(a_{i}^{j}\right)= \begin{cases}\left\lceil\frac{i+2 j-2}{2}\right\rceil, & j=1,2,1 \leq i \leq\left\lfloor\frac{j+3}{2}\right\rfloor \\ \left\lceil\frac{j^{2}+4 j+4 i-5}{8}\right\rceil, & j>1, \text { odd }, 1 \leq i \leq\left\lfloor\frac{j+3}{2}\right\rfloor \\ \left\lceil\frac{j^{2}+6 j-4 i+4}{8}\right\rceil, & j>2, \text { even }, 1 \leq i \leq\left\lfloor\frac{j+3}{2}\right\rfloor\end{cases}
$$

Now we evaluate weights for all edges as follows:

$$
w_{t}\left(a_{1}^{j} a_{1}^{j+1}\right)=\left\lceil\frac{j^{2}+6 j+4}{4}\right\rceil, 1 \leq j \leq n-1
$$




$$
w_{t}\left(a_{i}^{j} a_{i+1}^{j}\right)= \begin{cases}2, & i=1, j=1 \\ \frac{j^{2}+4 j+4 i-1}{4}, & j>1, \text { odd } ; \leq i \leq\left\lfloor\frac{j+1}{2}\right\rfloor \\ \frac{j^{2}+6 j-4 i+4}{4}, & j \geq 2, \text { even } ; \leq i \leq\left\lfloor\frac{j+1}{2}\right\rfloor\end{cases}
$$

On the basis of above calculations we see that all edges have distinct weights.

Hence, es $\left(C d_{n}\right)=\frac{n^{2}+6 n}{8}$

\section{Comb Graph $C e_{n}$ :}

Comb graph $C e_{n}$ is formed by vertex set $V\left(C e_{n}\right)=\left\{a_{i}^{j} ; 1 \leq j \leq n, 1 \leq\right.$ $i \leq 2 j+1\}$ and edge set $E\left(C e_{n}\right)=\left\{a_{i+1}^{j} a_{i+2}^{j+1} ; 1 \leq j \leq n-1, i=\right.$ $j\} \bigcup\left\{a_{i}^{j} a_{i+1}^{j} ; 1 \leq j \leq n, 1 \leq i \leq 2 j\right\}$. It has $n^{2}+2 n$ vertices and $n^{2}+2 n-1$ edges.

Theorem 5.1. Let $C e_{n}$ be a comb graph. Then es $\left(C e_{n}\right)=\left\lfloor\frac{(n+1)^{2}}{2}\right\rfloor$

Proof. Let $C e_{n}$ be a comb graph. We have to show that $e s\left(C e_{n}\right)=$ $\frac{(n+1)^{2}}{2}$. From Theorem 1.1 we get lower bound $e s\left(C e_{n}\right) \geq\left\lfloor\frac{(n+1)^{2}}{2}\right\rfloor$. For converse, we have to prove that $e s\left(C e_{n}\right) \leq\left\lfloor\frac{(n+1)^{2}}{2}\right\rfloor$. For this define a labeling on vertex set such that

$$
f\left(a_{i}^{j}\right)=\left\lceil\frac{j^{2}+i-1}{2}\right\rceil, \text { if } 1 \leq i \leq 2 j+1,1 \leq j \leq n
$$

Now we evaluate weights for all edges as follows:

$$
\begin{gathered}
w_{t}\left(a_{i+1}^{j} a_{i+2}^{j+1}\right)=(i+1)^{2}, \text { if } i=j, 1 \leq j \leq n-1 \\
w_{t}\left(a_{i}^{j} a_{i+1}^{j}\right)=i+j^{2}, \text { if } 1 \leq i \leq 2 j, 1 \leq j \leq n .
\end{gathered}
$$

On the basis of above calculations we see that all edges have distinct weights.

Hence, es $\left(C e_{n}\right)=\left\lfloor\frac{(n+1)^{2}}{2}\right\rfloor$ 


\section{Comb Graph $C f_{n}$ :}

Let $C f_{n}$ be a comb graph, which is formed by vertex set $V\left(C f_{n}\right)=\left\{a_{i}^{j} ; 1 \leq\right.$ $i \leq l, 1 \leq j \leq n\}$ and edge set $E\left(C f_{n}\right)=\left\{a_{\frac{l+1}{2}}^{j} a_{\frac{l+1}{2}}^{j+1} ; 1 \leq j \leq n-\right.$ $1\} \bigcup\left\{a_{i}^{j} a_{i+1}^{j} ; 1 \leq j \leq n, 1 \leq i \leq l-1\right\}$. It has $(n l)$ vertices and $(n l-1)$ edges and $l$ is the length of vertices in each $\mathrm{j}$

Theorem 6.1. Let $C f_{n}$ be a comb graph. Then es $\left(C f_{n}\right)=\left\lceil\frac{n l}{2}\right\rceil$

Proof. Let $C f_{n}$ be a comb graph. We have to show that $e s\left(C f_{n}\right)=$ $\left\lceil\frac{n l}{2}\right\rceil$. From Theorem 1.1 we get lower bound $e s\left(C f_{n}\right) \geq\left\lceil\frac{n l}{2}\right\rceil$. For converse, we have to prove that $e s\left(C f_{n}\right) \leq\left\lceil\frac{n l}{2}\right\rceil$. For this define a labeling on vertex set such that

$$
f\left(a_{i}^{j}\right)=\left\lceil\frac{l(j-1)+i}{2}\right\rceil 1 \leq i \leq l, 1 \leq j \leq n
$$

Now we evaluate weights for all edges as follows:

$$
\begin{gathered}
w_{t}\left(a_{\frac{l+1}{2}}^{j} a_{\frac{l+1}{2}}^{j+1}\right)=l j+1,1 \leq j \leq n-1 \\
w_{t}\left(a_{i}^{j} a_{i+1}^{j}\right)=i+l(j-1)+1,1 \leq i \leq l-1,1 \leq j \leq n
\end{gathered}
$$

On the basis of above calculations we see that all edges have distinct weights.

Hence, $e s\left(C f_{n}\right)=\left\lceil\frac{n l}{2}\right\rceil$

\section{Comb Graph $C g_{n}$ :}

Comb graph $C g_{n}$ is formed by vertex set $V\left(C g_{n}\right)=\left\{a_{i}^{j} ; 1 \leq i \leq j+1,1 \leq\right.$ $\left.j \leq\left\lceil\frac{n}{2}\right\rceil\right\} \bigcup\left\{a_{i}^{j} ;\left\lceil\frac{n}{2}\right\rceil<j \leq n, i=n-j+2\right\}$ and edge set $E\left(C g_{n}\right)=$ $\left\{a_{1}^{j} a_{1}^{j+1} ; 1 \leq j \leq n-1\right\} \bigcup\left\{a_{i}^{j} a_{i+1}^{j} ; 1 \leq j \leq\left\lceil\frac{n}{2}\right\rceil, 1 \leq i \leq j\right\} \bigcup\left\{a_{i}^{j} a_{i+1}^{j} ;\left\lceil\frac{n}{2}\right\rceil<\right.$ $j \leq n, i=n-j+1\}$. It has $\left(\frac{n^{2}+6 n+1}{4}\right)$ vertices and $\left(\frac{n^{2}+6 n-3}{4}\right)$ edges.

Theorem 7.1. Let $C g_{n}$ be a comb graph, then es $\left(C g_{n}\right)=\left\lfloor\frac{n^{2}+6 n+5}{8}\right\rfloor$ 
Proof. Let $C g_{n}$ be a comb graph. We have to show that $e s\left(C g_{n}\right)=$ $\left\lfloor\frac{n^{2}+6 n+5}{8}\right\rfloor$. From Theorem 1.1 we get lower bound $e s\left(C g_{n}\right) \geq\left\lfloor\frac{n^{2}+6 n+5}{8}\right\rfloor$. For converse, we have to prove that $e s\left(C g_{n}\right) \leq\left\lfloor\frac{n^{2}+6 n+5}{8}\right\rfloor$. For this define a labeling on vertex set such that

$$
f\left(a_{i}^{j}\right)= \begin{cases}\left\lceil\frac{i}{2}\right\rceil, & \text { if } i=1,2 ; j=1 \\ \left\lfloor\frac{j^{2}+2 j+2 i-1}{4}\right\rfloor, & \text { if } 1 \leq i \leq j+1, \text { odd } ; 2 \leq j \leq\left\lceil\frac{n}{2}\right\rceil \\ \left\lfloor\frac{j^{2}+2 i}{4}\right\rfloor, & \text { if } 2 \leq i \leq j+1, \text { even } ; 2 \leq j \leq\left\lceil\frac{n}{2}\right\rceil\end{cases}
$$

If $n \equiv 5(\bmod 4)$,
$f\left(a_{i}^{j}\right)= \begin{cases}\left\lfloor\frac{\left(\left\lceil\frac{n+2}{2}\right\rceil+1\right)^{2}}{4}\right\rfloor+\left\lfloor\frac{i}{2}\right\rfloor, & \text { if } j=\left\lceil\frac{n+2}{2}\right\rceil ; 1 \leq i \leq j-1, \\ \text { odd } & \\ \left\lfloor\frac{\left(\left\lceil\frac{n+2}{2}\right\rceil+1\right)^{2}}{4}\right\rfloor+\sum_{j>\left\lceil\frac{n+2}{2}\right\rceil}^{n}\left\lceil\frac{n-j+2}{2}\right\rceil+\left\lfloor\frac{i}{2}\right\rfloor, & \text { if }\left\lceil\frac{n+2}{2}\right\rceil<j \leq n ; \\ 1 \leq i \leq n-j+2, \text { odd } & \text { if } j=\left\lceil\frac{n+2}{2}\right\rceil ; 2 \leq i \leq j-1, \\ \left\lceil\frac{\left(\left\lceil\frac{n+2}{2}\right\rceil^{2}+3\right)}{4}\right\rceil+\frac{i-2}{2}, & \\ \text { even } & \\ \left\lceil\frac{\left(\left\lceil\frac{n+2}{2}\right\rceil^{2}+3\right)}{4}\right\rceil+\sum_{j>\left\lceil\frac{n+2}{2}\right\rceil}^{n}\left\lceil\frac{n-j+3}{2}\right\rceil+\frac{i-2}{2}, & \text { if }\left\lceil\frac{n+2}{2}\right\rceil<j \leq n ; \\ 2 \leq i \leq n-j+2, \text { even }\end{cases}$

$$
\begin{aligned}
& \text { If } n \equiv 3(\bmod 4) \text {, }
\end{aligned}
$$

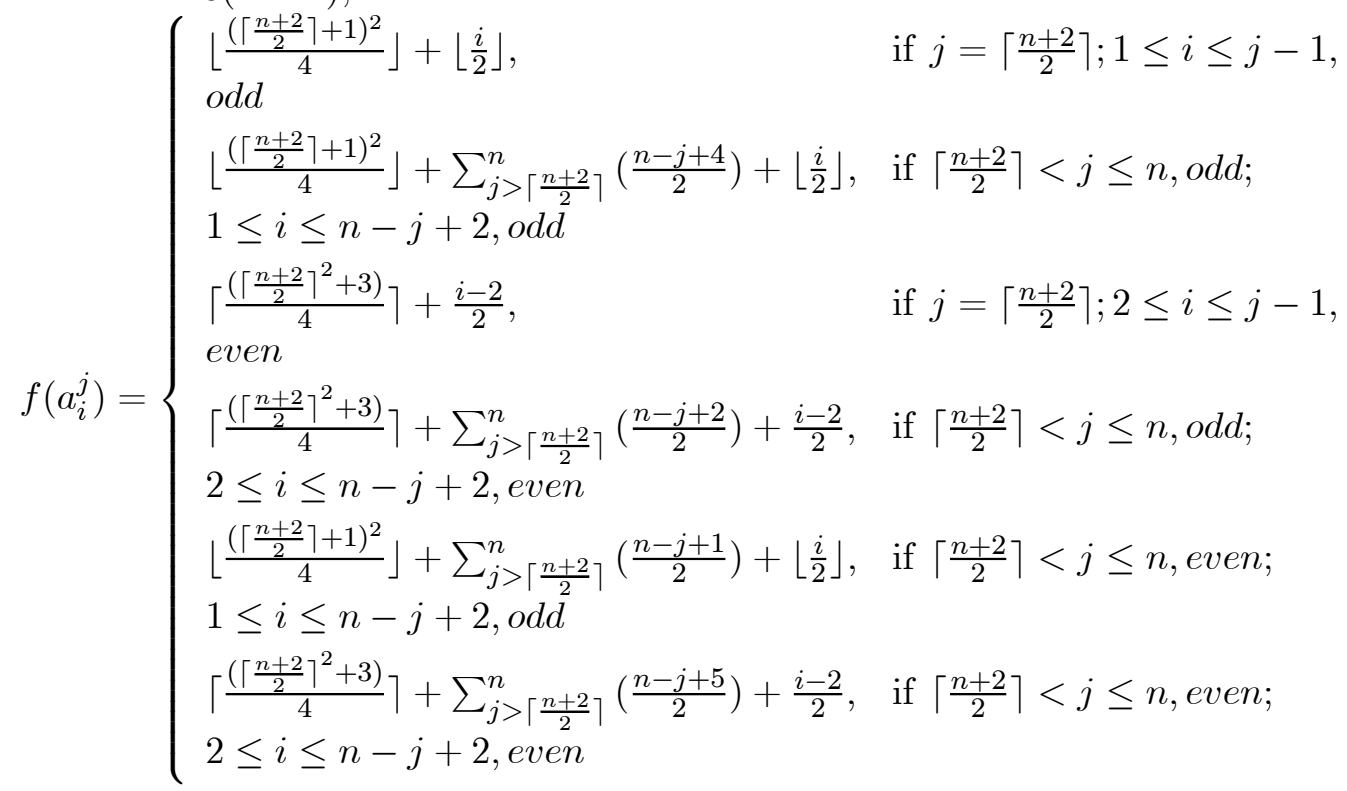


Now we evaluate weights for all edges as follows:

$$
\begin{gathered}
w_{t}\left(a_{i}^{j} a_{i+1}^{j}\right)=\frac{j^{2}+j+2 i}{2}, \text { if } 1 \leq j \leq\left\lceil\frac{n}{2}\right\rceil, 1 \leq i \leq j \\
w_{t}\left(a_{1}^{j} a_{1}^{j+1}\right)=\frac{j^{2}+3 j+2}{2}, \text { if } 1 \leq j \leq\left\lceil\frac{n}{2}\right\rceil
\end{gathered}
$$

If $n \equiv 5(\bmod 4)$

$$
w_{t}\left(a_{i}^{j} a_{i+1}^{j}\right)= \begin{cases}\alpha+\beta, & \text { if } j=\left\lceil\frac{n+2}{2}\right\rceil ; 1 \leq i \leq n-j+1 \\ \gamma+\delta, & \text { if }\left\lceil\frac{n+2}{2}\right\rceil<j \leq n ; 1 \leq i \leq n-j+1\end{cases}
$$

where

$$
\begin{gathered}
\alpha=\left\lfloor\frac{\left(\left\lceil\frac{n+2}{2}\right\rceil+1\right)^{2}}{4}\right\rfloor+\left\lfloor\frac{i}{2}\right\rfloor \\
\beta=\left\lceil\frac{\left(\left(\left\lceil\frac{n+2}{2}\right\rceil\right)^{2}+3\right)}{4}\right\rceil+\left\lfloor\frac{i-1}{2}\right\rfloor \\
\gamma=\left\lfloor\frac{\left(\left\lceil\frac{n+2}{2}\right\rceil+1\right)^{2}}{4}\right\rfloor+\sum_{j>\left\lceil\frac{n+2}{2}\right\rceil}^{n}\left\lceil\frac{n-j+2}{2}\right\rceil+\left\lfloor\frac{i}{2}\right\rfloor \\
\delta=\left\lceil\frac{\left(\left(\left\lceil\frac{n+2}{2}\right\rceil\right)^{2}+3\right)}{4}\right\rceil+\sum_{j>\left\lceil\frac{n+2}{2}\right\rceil}^{n}\left\lceil\frac{n-j+3}{2}\right\rceil+\left\lfloor\frac{i-1}{2}\right\rfloor .
\end{gathered}
$$

If $n \equiv 3(\bmod 4)$,

$$
w_{t}\left(a_{i}^{j} a_{i+1}^{j}\right)= \begin{cases}\alpha+\beta, & \text { if } j=\left\lceil\frac{n+2}{2}\right\rceil ; 1 \leq i \leq n-j+1 \\ \gamma+\delta, & \text { if }\left\lceil\frac{n+2}{2}\right\rceil<j \leq n, \text { odd } ; 1 \leq i \leq n-j+1 \\ \lambda+\mu, & \text { if }\left\lceil\frac{n+2}{2}\right\rceil<j \leq n, \text { even } ; \leq i \leq n-j+1\end{cases}
$$

where

$$
\begin{aligned}
& \alpha=\left\lfloor\frac{\left(\left\lceil\frac{n+2}{2}\right\rceil+1\right)^{2}}{4}\right\rfloor+\left\lfloor\frac{i}{2}\right\rfloor \\
& \beta=\left\lceil\frac{\left.\left(\left(\frac{n+2}{2}\right\rceil\right)^{2}+3\right)}{4}\right\rceil+\left\lfloor\frac{i-1}{2}\right\rfloor \\
& \gamma=\left\lfloor\frac{\left(\left\lceil\frac{n+2}{2}\right\rceil+1\right)^{2}}{4}\right\rfloor+\sum_{j>\left\lceil\frac{n+2}{2}\right\rceil}^{n}\left(\frac{n-j+4}{2}\right)+\left\lfloor\frac{i}{2}\right\rfloor \\
& \delta=\left\lceil\frac{\left(\left(\left\lceil\frac{n+2}{2}\right\rceil\right)^{2}+3\right)}{4}\right\rceil+\sum_{j>\left\lceil\frac{n+2}{2}\right\rceil}^{n}\left(\frac{n-j+2}{2}\right)+\left\lfloor\frac{i-1}{2}\right\rfloor \\
& \lambda=\left\lfloor\frac{\left(\left\lceil\frac{n+2}{2}\right\rceil+1\right)^{2}}{4}\right\rfloor+\sum_{j>\left\lceil\frac{n+2}{2}\right\rceil}^{n}\left(\frac{n-j+1}{2}\right)+\left\lfloor\frac{i}{2}\right\rfloor \\
& \mu=\left\lceil\frac{\left.\left(\left(\frac{n+2}{2}\right\rceil\right)^{2}+3\right)}{4}\right\rceil+\sum_{j>\left\lceil\frac{n+2}{2}\right\rceil}^{n}\left(\frac{n-j+5}{2}\right)+\left\lfloor\frac{i-1}{2}\right\rfloor
\end{aligned}
$$

On the basis of above calculations we see that all edges have distinct weights.

Hence we can say that $e s\left(C g_{n}\right)=\left\lfloor\frac{n^{2}+6 n+5}{8}\right\rfloor$ 


\section{Conclusion:}

In this paper, we obtained exact values of edge irregularity strength of different families of comb graph.

\section{References}

[1] A. Ahmad, M. Arshad, and G. Izarikova, "Irregular labelings of helm and sun graphs", AKCE international journal of graphs and combinatorics, vol. 12, no. 2-3, pp. 161-168, Nov. 2015, doi:10.1016/j.akcej.2015.11.010

[2] A. Ahmad, O. B. S. Al-Mushayt, and M. Baca, "On edge irregularity strength of graphs", Applied mathematics and computation, vol. 243, pp. 607-610, Sep. 2014, doi:10.1016/j.amc.2014. 06.028

[3] A. Ahmad, M. Bača, Y. Bashir, M. K. Siddiqui, "Total edge irregularity strength of strong product of two paths", Ars combinatoria, vol. 106, pp. 449-459, 2012.

[4] A. Ahmad, M. Bača, and M. K. Siddiqui, "On edge irregular total labeling of categorical product of two cycles", Theory of computing systems, vol. 54, no. 1, pp. 1-12, Apr. 2013, doi:10.1007/s00224-013-9470-3

[5] A. Ahmad, M. Bača, M., M. F. Nadeem, "On edge irregularity strength of Toeplitz graphs", Scientific Bulletin - "Politehnica" University of Bucharest. Series A, Applied mathematics and physics, vol. 78, no. 4, pp. 155162, 2016. [On line]. Available: https://bit.ly/2N3aeOZ

[6] O. B. S. Al-Mushayt, "On edge irregularity strength of products of certain families of graphs with path P-2", Ars combinatoria, vol. 135, pp. 323-334, 2017.

[7] O. B. S. Al-Mushayt, A. Ahmad, M. K. Siddiqui, "On the total edge irregularity strength of hexagonal grid graphs", Australasian journal combinatorics, vol. 53, pp. 263-272, Jun. 2012. [On line]. Available: https://bit.ly/2YGkbYf 
[8] D. Amar and 0. Togni, "Irregularity strength of trees", Discrete mathematics, vol. 190, no. 1-3, pp. 15-38, Aug. 1998, doi:10.1016/s0012$365 \times(98) 00112-5$

[9] M. Anholcer, M. Kalkowski, and J. Przybyo, "A new upper bound for the total vertex irregularity strength of graphs", Discrete mathematics, vol. 309, no. 21, pp. 6316-6317, Nov. 2009, doi: 10.1016/j.disc.2009.05.023

[10] M. Bača, S. Jendrol, M. Miller, and J. Ryan, "On irregular total labellings", Discrete mathematics, vol. 307, no. 11-12, pp. 1378-1388, May 2007, doi:10.1016/j.disc.2005.11.075

[11] M. Bača and M. K. Siddiqui, "Total edge irregularity strength of generalized prism", Applied mathematics and computation, vol. 235, pp. 168173, May 2014, doi:10.1016/j.amc.2014.03.001

[12] T. Bohman and D. Kravitz, "On the irregularity strength of trees", Journal of graph theory, vol. 45, no. 4, pp. 241-254, 2004, doi:10.1002/jgt.10158

[13] G. Chartrand, M. S. Jacobson, J. Lehel, O. R. Oellermann, S. Ruiz, F. Saba, "Irregular networks", Congressus numerantium, vol. 64, pp. 187-192, 1988.

[14] A. Frieze, R. J. Gould, M. Karoski, and F. Pfender, “On graph irregularity strength", Journal of graph theory, vol. 41, no. 2, pp. 120-137, Sep. 2002, doi:10.1002/jgt.10056

[15] K. M. M. Haque, "Irregular total labellings of generalized Petersen graphs", Theory of computing systems, vol. 50, no. 3, pp. 537-544, Jul. 2011, doi:10.1007/s00224-011-9350-7

[16] J. Ivanco and S. Jendrol, "Total edge irregularity strength of trees", Discussiones mathematicae graph theory, vol. 26, no. 3, pp. 449-456, 2006, doi: $10.7151 /$ dmgt.1337

[17] S. Jendrol, J. Miskuf, and R. Sotak, "Total edge irregularity strength of complete graphs and complete bipartite graphs", Discrete mathematics, vol. 310, no. 3, pp. 400-407, Feb. 2010, doi:10.1016/j.disc.2009.03.006 
[18] M. Kalkowski, M. Karonski, and F. Pfender, "A new upper bound for the irregularity strength of graphs", SIAM journal on discrete mathematics, vol. 25, no. 3, pp. 1319-1321, Jan. 2011, doi: $10.1137 / 090774112$

[19] J. Liu, J. Zhao, and Z. Zhu, "On the number of spanning trees and normalized Laplacian of linear octagonal quadrilateral networks", International journal of quantum chemistry, vol. 119, no. 17, May 2019, doi: 10.1002/qua.25971

[20] P. Majerski and J. Przybylo, "Total vertex irregularity strength of dense graphs", Journal of graph theory, vol. 76, no. 1, pp. 34-41, Jul. 2013, doi: $10.1002 /$ jgt.21748

[21] Nurdin, E. T. Baskoro, A. N. M. Salman, and N. N. Gaos, "On the total vertex irregularity strength of trees", Discrete mathematics, vol. 310, no. 21, pp. 3043-3048, Nov. 2010, doi: 10.1016/j.disc.2010.06.041

[22] J. Przybylo, "Linear bound on the irregularity strength and the total vertex irregularity strength of graphs", SIAM journal on discrete mathematics, vol. 23, no. 1, pp. 511-516, Jan. 2009, doi: $10.1137 / 070707385$

[23] I. Tarawneh, R. Hasni, and A. Ahmad, "On the edge irregularity strength of corona product of cycle with isolated vertices", AKCE international journal of graphs and combinatorics, vol. 13, no. 3, pp. 213-217, Dec. 2016, doi: 10.1016/j.akcej.2016.06.010

[24] H. Yang, M. A. Rashid, S. Ahmad, M. K. Siddiqui, and M. F. Hanif, "Cycle super magic labeling of pumpkin, octagonal and hexagonal graphs", Journal of discrete mathematical sciences and cryptography, vol. 22, no. 7, pp. 1165-1176, Oct. 2019, doi: 10.1080/09720529. 2019.1698800 